\title{
Competition between shear band nucleation and propagation across rate-dependent flow transitions in a model metallic glass
}

Matthew B Harris, Lars S Watts, Eric R Homer*

Department of Mechanical Engineering, Brigham Young University, Provo, UT 84602, USA.

\begin{abstract}
Shear transformation zone (STZ) dynamics is used to examine the transition between different regimes of flow serration in the strain rate dependent deformation of metallic glass. To capture the strain-rate independent yield strength of Vitreloy 1 at low to moderate strain rates, the model is adapted to include STZ volume and activation energy that decrease with increasing strain rate. The different stages of shear banding are examined in a statistical fashion over six different strain rates ranging from $10^{-5}$ to $10^{0} \mathrm{~s}^{-1}$, with 12 replicates at each strain rate. Examination of flow serration, shear band nucleation rates, propagation rates, and sliding rates in each simulation find support for the hypothesis that the flow transition is caused by fast shear band propagation and sliding rates at low strain rates, and fast shear band nucleation rates at high strain rates. The underlying cause for the flow transition is hypothesized to be a strain rate dependent critical shear band nucleus size that increases with increasing strain rate. This critical shear band nucleus size results from the strain rate dependent STZ volume and activation energy, from which very small variations cause a large change in shear banding behavior.
\end{abstract}

Keywords: metallic glass; shear transformation zone; modeling; flow serration; shear band

*Corresponding Author: eric.homer@byu.edu

(C) 2016. This manuscript version is made available under the Elsevier user license http://www.elsevier.com/open-access/userlicense/1.0/ 


\section{Introduction}

Metallic glasses show great promise as lightweight, high strength, flexible materials due to their impressive mechanical properties [1-3]. However, metallic glasses suffer from poor ductility at room temperature due to their tendency to localize plastic strain into shear bands $[2,4,5]$, which ultimately lead to catastrophic failure. Interestingly, although the yield point of these materials is often independent of strain rate for rates up to $10^{2}-10^{3} \mathrm{~s}^{-1}$, the shear band density and degree of flow serration are highly strain rate dependent [6-8]. A thorough understanding of the mechanisms underlying this phenomena is necessary to enable the development of tougher, more ductile metallic glass composites and alloys.

The different modes of deformation, homogeneous and inhomogeneous, exhibited by metallic glasses are well characterized by examination of Schuh's deformation map, shown in Figure 1 [6]. The homogeneous regime exists at elevated temperatures and lower strain rates, where the deformation is characterized by viscous flow. The inhomogeneous regime is characterized by localized deformation, typically in the form of shear bands. This regime encompasses temperatures below the glass transition temperature $\left(T_{g}\right)$ at lower strain rates to a much larger temperature range at higher strain rates. Within the inhomogeneous regime, deformation at low strain rates is characterized by strongly serrated flow, meaning that strain accumulates in the material in temporal bursts accompanied by relaxation stress drops resulting in a jagged stress-strain curve $[9,10]$. Higher strain rates are characterized by moderately serrated flow, and very high strain rates have little or no flow serration. In nanoindentation experiments, Schuh and Jiang independently observed that this reduced flow serration was accompanied by a reduction in the appearance of shear steps in the surface of the material around the indenter $[11,12]$. At the lower strain rates, the plasticity is localized into only a few shear bands, at higher strain rates the plasticity is dispersed across many shear bands. It has been hypothesized that this 
change from few to many shear bands at increasing strain rate is due to competition between shear band nucleation and propagation [7]; when individual shear bands nucleate and propagate quickly relative to the strain rate, the stress in the surrounding material is reduced, suppressing additional shear band nucleation. However, when shear bands do not accommodate strain quickly enough to relieve stress in the material, multiple shear bands occur to reduce the stress.

The process by which individual shear bands nucleate, propagate, and arrest has been the subject of continued investigation. The explanation of this process begins with the fundamental unit of deformation in metallic glasses, which is accepted to conform to the shear transformation zone (STZ) theory introduced by Argon [13]. The STZ involves the collective and inelastic rearrangement of several dozen atoms in response to an applied shear stress. The action of one STZ causes an increase in the local stress field along the direction of shear, creating preferential sites for the activation of additional STZs $[14,15]$. Models applying Argon's STZ theory have differed on how shear bands form and propagate from an initial group of STZs, with three main viewpoints being most prominent. First, some model the shear band as a percolating boundary that reaches a critical concentration of STZs before initiating simultaneous slip along the plane of highest resolved shear stress [16]. Second, shear bands are modeled as a propagating zone of rejuvenated glass, followed by a zone of glue-like material, and finally followed by liquid material, as adiabatic heating decreases the local strength [17]. Third, others model a two-step process, with a shear band nucleating from a small cluster of STZs, and propagating quickly through the sample before initiating simultaneous slip $[18,19]$. Recent work by Qu, et al. has shown that metallic glass samples pulled to very low levels of plastic strain show signs of partially propagated shear bands [20], lending further credibility to the second and third theories. The two-step theory, as explained by Homer, Schuh, Greer and others $[6,15,18,19,21]$, can be subdivided into three stages for the progression of deformation in metallic glasses:

1. Nucleation: STZs activate, cluster, and make up the growing nuclei of competing shear bands 
2. Propagation: When a shear band nucleus reaches a critical size, it begins to rapidly grow, dominating plasticity in the region

3. Sliding: Stress relaxation occurs as the fully developed shear band thickens and accumulates additional plasticity as shear band slip, until the applied load decreases enough for slip to arrest

Once a shear band has arrested, the free volume generated by the action of STZs remains, and allows it to be preferentially reactivated [22-24]. In other works, stages 1 and 2 are generally referred to as shear band initiation, while stage 3 is referred to as shear band propagation. In this paper, shear bands are analyzed for their progression through all 3 stages. Stage 1, nucleation, ends when a shear band becomes dominant, stage 2 continues until the shear band reaches the full width of the simulation, and stage 3, sliding, encompasses all plasticity that takes place on the band after it is fully propagated.

Investigating the transition between different flow serration regimes requires a collection of shear band events to be studied in a statistical manner so an understanding can be gained of how the mechanics of shear band formation influence flow serration. Researchers have used several different approaches to resolve shear band events in experimental setups. For example, high speed cameras have been able to capture shear band sliding, and measure shear band velocities [25]. They also show that flow serration is often the result of the same shear band being activated multiple times, rather than unique shear bands for each event [22]. Analysis of pop-in stresses during nanoindentation enabled estimation of STZ volumes and rate effects $[26,27]$. Although this information is very useful, such experimental methods are unable to reveal the details of what is happening at the STZ level in shear band nucleation and propagation; the time and length scales of individual STZs are too small and fast for current measurement resolutions to capture directly, and indirect measurements do not give a complete picture.

Modeling techniques provide unique insight into the possible processes of shear band formation. Atomistic simulations do well at simulating the action of individual STZs, capturing the onset of shear localization in metallic glass [28]. They can measure the STZ volume for various glass compositions, and 
have shown that the instability of shear bands arises from structural disordering in an STZ, rather than thermal softening $[19,29]$. Constitutive models do well at recreating the macroscopic behavior of metallic glass. By treating the glass as a continuum material, rather than trying to simulate each individual atom or STZ, they enable more complicated structures and loads to be modeled, within the limits of the constitutive model's scope [30,31]. Mesoscale models are needed to investigate the range of time and length scales intermediate to molecular dynamics and constitutive models [28]. One such mesoscale model is the STZ dynamics model developed by Homer and Schuh [32]. The STZ dynamics model is able to capture a broad range of time scales associated with shear band events in an efficient manner by using a kinetic Monte Carlo algorithm [14]. It has been used to simulate both 2D and 3D metallic glass structures; it predicts a propagating shear band, and captures the transition from inhomogeneous to homogeneous flow at the glass transition temperature [21, 33]. It has even been adapted by Li to account for free volume generation due to STZ activity [34]. Since this model is capable of simulating the nucleation and growth of multiple shear bands, and these are the parameters of interest, we use the STZ dynamics model for our investigation of flow serration regimes in metallic glasses.

In this work, the STZ dynamics model is adjusted to maintain a constant yield point over strain rates of $10^{-5}-10^{0} \mathrm{~s}^{-1}$, consistent with the behavior of Vitreloy 1 , and other metallic glasses $[6,8,35]$. With the adjusted model parameters, we examine flow serration and the early stages of shear band nucleation and propagation across a range of strain rates, with multiple simulations at each strain rate to determine statistical variance. Discussion of the results shows support for the hypothesis of competing shear band nucleation and propagation rates, and is focused on determining the underlying causes of this interaction. A hypothesis is developed to explain the simulated behaviors, and its implications are explored in the conclusion. 


\section{Methods}

\subsection{STZ dynamics modeling framework}

The STZ dynamics model is built around the STZ as the fundamental unit of deformation in metallic glass. The STZs are coarse-grained by replacing groups of atoms with the features of a finite-element mesh. Each node in the mesh represents the center of a potential STZ, and the five to seven elements touching that node represent the atoms which collectively shear if that STZ is activated [32]. Each element can participate in different STZs, just as atoms may participate in various STZs in a real material. These course-grained STZs are treated mathematically as Eshelby inclusions with coherent boundaries, the same approach originally used by Argon, where the STZ is allowed to plastically deform as if in a vacuum and then forced elastically back into the surrounding matrix [13]. The kinetic Monte Carlo (kMC) algorithm is used to control the evolution of the modeling framework. When a given STZ is selected for shearing by the $\mathrm{kMC}$ algorithm, plastic strains are applied to the elements to simulate the simultaneous plastic shearing of atoms in the STZ. After each KMC step, finite-element analysis solves for the resulting stress and strain fields throughout the simulation, which then influence the selection of STZs in subsequent kMC steps [32].

The kMC algorithm works by listing all the possible transitions a system can make, calculating each transition's associated rate, and then using a random number to select one of the transitions for execution. Time in the simulation is then advanced by $\Delta t$ based on the residence time in the current configuration, which is also based on the rates of the possible transitions. A more complete description of the kMC algorithm is available from Voter [36]. In STZ dynamics, a modified kMC algorithm, introduced previously [14], is used to ensure that realistic times and transitions are represented. This is accomplished by suppressing any STZ events selected by the algorithm which would activate in a $\Delta t$

greater than a certain maximum allowed time step $\left(\Delta t_{\text {max }}\right)$. When this happens, the model increments 
time by $\Delta t_{\text {max }}$ without activating any STZ. This allows other time-dependent parameters, such as stress and strain, to update on a reasonable time scale in a dynamic simulation beginning at very low stresses. After every time increment, whether an STZ is activated or not, finite element analysis (FEA) solves for the new stress distribution in the mesh, and new activation rates are calculated for use in the kMC algorithm.

The kMC algorithm requires a rate calculation for each possible event in its catalogue for selection. For the STZ dynamics framework, this is an STZ activation rate that calculates the rate at which a given STZ will transition from an unsheared to a sheared state. The activation rate $\dot{s}$ for shearing a particular STZ in one direction is given by:

$$
\dot{S}=v_{0} \exp \left(-\frac{\Delta F-\frac{1}{2} \tau \gamma_{0} \Omega_{0}}{k T}\right)
$$

where $v_{0}$ is the attempt frequency (related to the Debye frequency), $\Delta F$ is the set activation energy barrier for shearing an STZ, $T$ is the temperature in Kelvin, $k$ is Boltzmann's constant, $\tau$ is the local shear stress in the direction of shear, $\gamma_{0}$ is the incremental shear strain applied to an STZ, and $\Omega_{0}$ is the volume of an STZ. This rate captures the thermally activated nature of the shearing process, which can be biased by both its local shear stress $\tau$ and the temperature T. A more detailed explanation of this equation's parameters as they relate to the STZ dynamics model can be found in previous work [32].

The coarse-graining of the STZs follows previous works where node-centered STZs are defined on a uniquely generated, irregular, triangular mesh [32]. The mesh size is defined such that the average radius of the potential STZs is equal to the target STZ radius. Each simulation is generated with a length of $250 \mathrm{~nm}$ along the tensile axis, and a width of $50 \mathrm{~nm}$. The long sides of the simulation are unconstrained, and the top and bottom surfaces of the simulation are allowed to move laterally relative to each other to enable lateral slip. The simulated tensile test is displacement controlled; this is achieved by constraining the bottom nodes, while the top nodes are displaced at a fixed velocity corresponding to 
the desired initial strain rate. In each step of the simulation, the nodes move in varying increments of strain corresponding to the elapsed time from the modified kMC algorithm.

The simulations are run at six different initial strain rates: $10^{-5}, 10^{-4}, 10^{-3}, 10^{-2}, 10^{-1}$, and $10^{0} \mathrm{~s}^{-1}$. With the simulation size of $250 \mathrm{~nm}$, this results in displacement velocities that range from 0.0025 to $250 \mathrm{~nm} / \mathrm{s}$, at the lowest and highest strain rates, respectively. Each simulation is run for as many kMC steps as necessary for the simulation to reach $1.9 \%$ total strain, regardless of strain rate. The maximum time step $\Delta t_{\max }$ values for the modified KMC algorithm are set at $5 \mathrm{~s}, 1 \mathrm{~s}, 0.5 \mathrm{~s}, 0.05 \mathrm{~s}, 0.005 \mathrm{~s}$, and $0.0005 \mathrm{~s}$, increasing from the longest time at the lowest strain rate to the shortest time at the highest strain rate. The variation in $\Delta t_{\text {max }}$ from one strain rate to the next is necessary to provide the needed time resolution that stabilizes the yield strength in each strain rate, balanced against a reasonable number of kMC steps for a given simulation. As an extreme example: at the $10^{0} \mathrm{~s}^{-1}$ strain rate, a large $\Delta t_{\text {max }}$ like $1 \mathrm{~s}$ would cause $100 \%$ strain in the first KMC step, before any STZs are able to activate. On the opposite end, a small $\Delta t_{\max }$ such as $.001 \mathrm{~s}$ used on the $10^{-5} \mathrm{~s}^{-1}$ strain rate, would require $10^{6}$ simulation steps to reach $1 \%$ strain, still short of yield. A representative strain rate was tested with several different values for $\Delta t_{\max }$ spanning two orders of magnitude, and it is found that this parameter has no noticeable effect on yield strength or STZ behavior, other than to overshoot the yield strength when $\Delta t_{\text {max }}$ is too large. Material property values used in the simulation correspond to those of Vitreloy 1 , a commonly studied metallic glass, listed in Table $1 ; \Delta F$ and $\Omega_{o}$ are defined in the following section. The simulation temperature is held constant at $310 \mathrm{~K}$, which is $0.5 T_{g}$ for Vitreloy 1.

\subsection{Parameterization of strain rate dependence}

The STZ dynamics model relies on the rate equation of STZ activation (Eq. 1) to control the evolution of the simulation and, as a result, it has an inherent strain rate dependence that leads to an increase in yield strength at higher strain rates. This is accompanied by increased shear band density at higher 
strain rates, which corresponds with the flow serration being studied. As noted above, most metallic glasses do not exhibit a strain rate dependent yield strength over the rates studied in this work [6, 35]; as a result, the STZ dynamics model must be adjusted to capture this behavior. The modifications to the STZ dynamics model are inspired by work by Dubach et al. and Tönnies et al., who find strain rate and temperature dependencies to the STZ volume [27, 30]; in some cases the STZ volume has a log-linear dependence on strain rate. As a result of this insight into the influence of STZ behaviors under differing conditions, it was determined that this dependence be included in the STZ dynamics framework.

In examining the influence of different model parameters one can show that increasing STZ volume $\left(\Omega_{0}\right)$ alone has the effect of decreasing the shear band density and lowering the yield strength. Increasing activation energy $(\Delta \mathrm{F})$ alone has no effect on shear band density, but increases the yield strength significantly. Thus, having $\Omega_{0}$ and $\Delta \mathrm{F}$ both decrease with increasing strain rate lessens the strain rate dependence of the yield strength while magnifying the increased shear band density at higher strain rates typical to metallic glasses. These are given by the following log-linear forms:

$$
\begin{gathered}
\Omega_{0}=-0.03772 \log _{10} \dot{\epsilon}+1.6\left[\mathrm{~nm}^{3}\right] \\
\Delta F=-0.02274 \log _{10} \dot{\epsilon}+1.07945[\mathrm{eV}]
\end{gathered}
$$

The STZ volume increases by $0.2 \mathrm{~nm}^{3}$ for each order of magnitude change in strain rate, ranging from 2.6 to $1.6 \mathrm{~nm}^{3}$, at the lowest and highest strain rates examined in this work, respectively. These values are selected to be consistent with STZ volumes found generally [15, 27, 37], and particularly with the size range found by Dubach et al. in their low temperature data [30]. The magnitude of $\Delta F$ across the strain rates ranges from $1.08 \mathrm{eV}$ to $1.68 \mathrm{eV}$, at the highest and lowest strain rates examined in this work, respectively. This represents a relatively minor variation compared to reported values of the activation energy [38, 39]. This solution is non-unique, as different forms for the $\Omega_{0}$ relation could be fit by a similar $\Delta F$ relation to give similar results, though these might in turn be non-log-linear, which is the form Dubach et al. report [30]. More importantly, a $\Omega_{0}$ relation with a positive slope reverses the flow 
serration behavior (i.e. high strain rates give low shear band density and low strain rates give high shear band density). As a result, there appears to be a physical basis behind the negative dependence of $\Omega_{0}$ on strain rate. For the few metallic glasses that do exhibit a strain rate dependent yield strength at low strain rates, such as the Zr-based metallic glass investigated in the work of Chen and Tsai [40], a modification to the STZ volume and activation energy, very similar to Eq. 2, would still be required to match the experimental data. Thus, it is expected that a model metallic glass with a strain rate dependent yield strength, would exhibit similar results to those reported here. Possible physical causes behind this strain-rate dependence on STZ volume and activation energy are considered in the discussion.

Figure 2 shows the results of the parameterization of strain rate dependence on the observed yield strength. Before fitting, there is a clear log-linear relationship between yield strength and strain rate, where a logarithmic fit has an $\mathrm{R}^{2}$ value of 0.9625 . After parameterizing $\Omega_{0}$ and $\Delta F$ to strain rate, however, the $R^{2}$ of the logarithmic fit of the yield strengths is reduced to 0.0271 , meaning that the logarithmic fit of the yield strength no longer describes the relationship significantly better than the arithmetic mean; any variation is now ascribed to random noise in the data. Thus, the parameterization is successful in eliminating the model's yield strength dependence on strain rate.

\subsection{Statistical analysis approach}

The goal of this work is to understand the mechanisms underlying the transitions between different regimes of flow serration in the deformation map. Emphasis is placed on measuring shear band nucleation and propagation rates since it is believed that different degrees of flow serration arise due to competition between these rates. In order to ensure statistical significance, twelve simulations are run at each of the six strain rates simulated, for a total of 72 simulations. At completion, the shear bands of each simulation are identified, as shown in Figures $3(a)$ and $3(b)$, where Figure $3(b)$ has each individual 
band highlighted in a unique color. These surrounding lines are placed carefully as they define which STZs contribute to each shear band, which is important for subsequent calculations. As a rule, any group of five or more STZs in a line is identified. Once the shear bands are identified, statistics are collected for:

1. The number of shear band nuclei in each simulation,

2. The dominance of individual shear bands during deformation,

3. Critical nucleus size of the first shear band when it becomes dominant,

4. Front propagation rate of each shear band,

5. Sliding velocity of the most dominant shear band once it has propagated across the entire simulation cell, and

6. The number and magnitude of stress drops in the simulation, which are used to measure the degree of flow serration in the simulation.

The manner in which each of these measurements is obtained is explained below, and demonstrated with an example simulation run at $10^{-4} \mathrm{~s}^{-1}$, shown in Figure 3.

The number of shear band nuclei in each simulation is obtained by counting the number of shear bands identified since each one must have started through the formation of a nucleus.

The measurement of the critical nucleus size of a shear band requires knowledge of when an individual shear band has reached a critical size and accelerates its growth, thereby dominating deformation in that moment. As such, we introduce an additional measurement called shear band dominance, which is measured by examining a simulation using a moving window of twenty kMC steps. In each temporal window, the number of STZs activated in each shear band is totaled. Shear band dominance is defined as the fraction of STZs activated in a given shear band for that window in time. This window is moved in two KMC step increments to determine shear band dominance over the evolution of the whole simulation. This is plotted against time in Figure 3(c), with the colors in the plot matching the coloring of the shear band selection lines in Figure 3(b). A window can contain fewer than twenty activated STZs if some of the KMC steps do not produce STZ activations; this generally happens during the initial loading of the simulation, where the model is accumulating elastic strain. To prevent hypersensitivity to 
windows that contain very few STZ activations, no shear band can be dominant if that twenty kMC step window does not contain at least ten STZ activations. This has several implications; one is that when very few STZs are active, it is possible that the sum of STZ activity in the plot will amount to less than $100 \%$. Similarly, some STZs in the simulation fall where two shear bands intersect; these STZs will count towards STZ activity in both shear bands and the sum of STZ activity can exceed $100 \%$. Finally, even if all of the first twenty STZs in a simulation fall on the same shear band, the dominance ramps up to $100 \%$ rather than being instantly $100 \%$ dominant.

Shear band critical nucleus size is then defined as the total volume of STZs in a shear band nucleus at the point in time where it reaches a critical level of shear band dominance. A threshold of $60 \%$ dominance is chosen as the proportion of STZ activity required for a shear band to be considered critical. In combination with the lower bound of ten STZs as the minimum number to be considered in calculating dominance, this means that the minimum critical shear band nucleus size that can be detected consists of six STZs. In this example simulation, the shear band marked with blue becomes dominant first, followed by the teal and magenta shear bands in later stages of the simulation. The shear bands marked with red and green never reach the threshold level of dominance. The critical nucleus sizes for each of these shear bands can be measured as their size when they become dominant, but for the purposes of this work, we examine only the first dominant shear band. This enables cleaner comparison in sizes between simulations and less variability in measurements due to shear band intersections later in the simulation.

Shear band propagation rate is measured by carefully finding the change in length of each shear band in a moving window of twenty $\mathrm{kMC}$ steps, then dividing that by the change in time for those twenty steps to give a velocity measurement. The evolution of the propagation speed is obtained by moving the window by two steps for each data point. The results of this measurement are plotted in Figure 3(d), with the same coloring scheme as in other parts of the figure. 
Shear band sliding rate is defined as the relative velocity between the part of the mesh just above and just below the shear band. This is calculated by selecting several points on the shear band selection lines above and below a given shear band. The relative velocities of the nodes in the sliding direction is calculated using the same moving window used in previous calculations, and the results are given in Figure 3(e). Shear band sliding rate is measured only for the thickest, most dominant shear band in each simulation. This is done to reduce the amount of interference from shear band intersection and for clearer comparison between different simulations.

In order to measure flow serration, stress drops in each simulation are found by measuring the slope of the stress strain curve as shown in Figure 3(f). It is noted that the three stress drops in Figure 3(f) correspond to repeated activation of the same shear band, as shown by Figure 3(g). When the slope is large and negative, (a drop of at least $5 \mathrm{MPa}$ in less than .000001 strain, for example) a stress drop is identified, with the value of the stress before and after the region of steep slope determining the magnitude of the drop as in [41]. Slopes are calculated in a moving window of twelve kMC steps, in order to prevent very small drops from dragging down average drop sizes. The average magnitude of these stress drops is used as a measure of flow serration in the simulation.

It is noted that the present work differs from those generally used in experimental setups measuring flow serration, where flow serration is characterized by strain bursts. For example, the stress drops in Figure $3(f)$ do not have the appearance of serrated flow about a constant flow stress for a sample in compression [9]. However, an experimental tensile test of a nanowire produces a stress-strain curve that is very similar to those shown in the results of this work, which are also of nanoscale sizes [42]. As such, the differences in flow serration examined here are attributed to differences in size of the specimens as compared with traditional flow serration in compression tests. 


\section{Results}

Figure 4 (a) shows a group of six simulations, one from each strain rate studied, with increasing strain rate from left to right. The simulations shown represent typical results, with the number of shear bands in each one near the median value for its strain rate. Each simulation is shown at the final strain value of 1.9\%. In general, low strain rates result in fewer, more dominant shear bands, with very few free STZs scattered outside the bands, while high strain rates feature larger numbers of less dominant shear bands, with many free STZs randomly scattered outside the bands. The stress strain curves for these six simulations are shown in Figure 4 (b), showing a tightly grouped yield strength around $1.72 \mathrm{GPa}$. While the yield strengths are similar, low strain rates tend to relax more quickly after yield, and have a lower flow stress than high strain rates.

\subsection{Flow serration}

The average magnitude of stress drops in each simulation is measured as discussed in section 2.3. These average stress drops are then categorized and summarized by strain rate using box plots in Figure 5 . These box plots mark the median value with the horizontal (red) line inside the box, the upper and lower quartiles of the distribution bound the box, and the maximum and minimum values are marked by whiskers that extend from the top and bottom of the box. Statistical outliers are marked as ' + ' in red. It can be seen that the average stress drop statistics have a negative correlation with strain rate. Strain rates in the Schuh's strong flow serration regime $\left(10^{-5}\right.$ to $\left.10^{-3} \mathrm{~s}^{-1}\right)$ have median values of $20-30 \mathrm{MPa}$, while those in the Schuh's moderate serration regime $\left(10^{-2}\right.$ to $\left.10^{0} \mathrm{~s}^{-1}\right)$ have values of 7-15 MPa. There is a marked decrease at $10^{-2} \mathrm{~s}^{-1}$, which is near the border of the transition from strong serration to light serration in Schuh's deformation map. The $10^{-2} \mathrm{~s}^{-1}$ strain rate also shows mixed behavior, with a few outlying simulations above the $20 \mathrm{MPa}$ range. To understand how this change in flow serration is 
influenced by the mechanics of STZ activation, we now examine the statistics of shear band nucleation and propagation rates.

\subsection{Shear band nucleation statistics}

Figure 6 provides statistics on the number of shear band nuclei, and the critical shear band nucleus size. Simulations at high strain rates show an increase in the number of shear band nuclei generated in a given simulation, as indicated by Figure 6(a). One can calculate the rate of shear band nucleation, in units of shear band nuclei generated per second, which is provided in the inset to Figure 6(a). This raw shear band nucleation rate is proportional to the strain rate raised to the power of $1.1\left(\epsilon^{1.1}\right)$, which is indicated by the overlaid power-law fit in the inset to Figure 6(a). However, the nucleation rate isn't necessarily the best comparison between different strain rates because the tests last different time periods. As such, the total number of nuclei (which is equivalent to nuclei per $1.9 \%$ strain), is the better comparison between strain rates. It is noted that the shear band nucleation rate has a positive correlation with strain rate, because the 1.1 proportionality exponent is greater than 1 . At low strain rates in the strong serration regime, a median of 4.5 to 6.5 shear band nuclei appeared throughout the entire simulation. At $10^{-2}$ strain/s and above this increases steadily, up to a median of 15.5 nuclei for the highest strain rate.

The statistics of the critical nucleus volume, or volume of the first shear band when it became dominant, is plotted in Figure 6(b). This critical volume has a positive correlation with strain rate. It is noted that at the highest strain rate, some of the simulations never have a shear band become dominant at all, resulting in fewer data points and an underestimation of the critical nucleus volume at that strain rate. Also, due to the fact that a lower bound is enforced on the measurement of critical shear band size, shear bands can never be considered dominant with fewer than six STZs. In Figure 6(b), we see that all strain rates from $10^{-5}$ to $10^{-2} \mathrm{~s}^{-1}$ have at least one simulation where the first shear band becomes 
dominant at exactly this lower bound. Thus, the lower bound may overestimate the critical nucleus size for these slower strain rates. At the lowest strain rate, two or three STZs may be enough to reach a critical volume for the shear band to nucleate and propagate unconstrained.

\subsection{Shear band propagation and sliding}

The statistics of the maximum propagation rate, or maximum speed of the shear band's propagating front, are presented as a rate, normalized by the strain rate in Figure 7(a). The unnormalized propagating speed is provided in the inset to Figure 7(a). Here it can be seen that before normalization, the maximum propagation speed of a growing shear band in a simulation at first seems to increase proportionally with the strain rate, though the proportionality between the propagation speed and the strain rate is exponential to the power of $0.56\left(\epsilon^{0.56}\right)$, which is indicated by the overlaid power-law fit in the inset to Figure 7(a). However, the normalization by the applied strain rate indicates that at higher strain rates, the relative maximum propagation rate actually decreases with increasing applied strain rate, because the proportionality exponent 0.6 is less than 1 . This means that while a single shear band grows faster at increasing strain rates, it is moving slower relative to the applied strain rate as that strain rate increases.

A similar relationship is found when looking at the maximum shear band sliding speed, which is shown in its strain rate normalized form in Figure $7(b)$. The unnormalized sliding speed is provided as an inset to Figure $7(b)$, which has a proportionality exponent of $0.59\left(\epsilon^{0.59}\right)$, as indicated by the overlaid power-law fit. Once again, the unnormalized form goes from a positive correlation with strain rate to a negative correlation when it is normalized by the applied strain rate, because the proportionality exponent is again less than 1 . This means that continued sliding of the largest shear band accounts for less and less of the total plasticity as the strain rate increases. By comparing Figure 7(a) to Figure 7(b), we also see that the normalized shear band propagation speed is about three orders of magnitude faster than the 
normalized shear band sliding speed, which is consistent with measurements related to the two-step shear band formation model, which place shear band propagation on the microsecond time scale and shear band sliding on the millisecond time scale [10].

In order to see how strain accumulates on the largest shear band over time, the shear band sliding speed is plotted against time for each simulation. Representatives of the highest and lowest strain rates are shown in Figure 8, with each plot appearing next to its respective simulation result. These simulations are chosen because each has a thick, dominant shear band, though these were rare at the highest strain rate. The graphs show that at the lowest strain rate, the dominant shear band accumulates plasticity in very sharp temporal bursts, with very little activity on the band in between these strain bursts. At the highest strain rate, even when the plasticity is concentrated mostly into a single band, the band accumulates plasticity in a more continuous manner with less pronounced temporal bursts. This is illustrative of the flow serrations expected for the two strain rates, indicating that the model is producing the expected behaviors.

\section{Discussion}

One important attribute of the STZ dynamics model is that the stochastic approach enables variation in a given process to be studied from identical starting simulation parameters. This variability partially fulfills Greer's suggestions for treating STZ behavior by statistical means [15]. In Figure 5, for example, at $10^{-2} \mathrm{~s}^{-1}$ most simulations belong in the regime of moderate flow serration, while a few outliers seem to belong in the strong flow serration regime [7]. Similarly, in Figure $6(\mathrm{~b})$, some of the simulations at $10^{0} \mathrm{~s}^{-1}$ never ended up nucleating a dominant shear band, which can mean that they match Schuh's transition from light, to little or no flow serration in the deformation map [7]. Such variability near regime changes in the deformation map, where a given sample could exhibit behavior from either regime, could be expected in experimental results. Thus, the model appears to be capturing transitions between distinct 
regimes, rather than simply modeling a general trend of decreasing flow serration with increasing strain rate.

A summary of the different statistics and their strain rate dependence is shown in Figure 9, where each rate has been scaled so they can be compared side by side. This shows a transition from shear band propagation-driven plasticity that dominates at low strain rates, to shear band nucleation-driven plasticity that dominates at high strain rates. This shows strong support for the hypothesis Schuh proposed, that the decreased flow serration seen at higher strain rates is due to the nucleation of many small shear bands in direct competition to the propagation of individual bands [11]. However, the underlying cause for this transition is not clear. One could question whether propagation is limiting nucleation, or vice versa.

We hypothesize that the critical shear band nucleus size is an underlying cause of the transition in flow serration. This is best illustrated by examining the stages of shear banding. In stage 1, STZs appear, and begin to cluster into shear band nuclei, which grow and proliferate in the absence of a dominant shear band. If the strain rate is low, then a small critical nucleus size means that stage 2 is reached quickly, and one shear band rapidly propagates across the sample, and begins to dominate all plasticity in the sample. Then, in stage 3 , additional plasticity is concentrated in bursts on that dominant band. If instead the strain rate is high, then a large critical nucleus size means that stage 2 is delayed, or skipped entirely, and plasticity continues to be accommodated by nucleation of additional shear band nuclei in stage 1. Then sliding in stage 3 occurs more gradually as shear band nuclei begin to intersect each other, and plasticity remains relatively diffuse.

In essence, this strain rate dependent critical shear band nucleus would be a criterion for a kinetic theory of shear band nucleation. This kinetic effect is important because a recent thermodynamic model of nucleation only predicts a critical stress for nucleation [21]. The thermodynamic model indicates very 
little dependence on nucleation size and does not predict the flow serration transitions evident in metallic glasses. Together, these kinetic and thermodynamic nucleation criteria provide a better picture of what is necessary to nucleate a shear band.

Speculating that critical shear band nucleus size is indeed the controlling factor indicates that the parameterization of STZ volume and STZ energy barrier as a function of strain rate may capture physical mechanisms of STZs. As noted earlier, experiments do indicate a STZ size dependence on strain rate [27, 30]. For example, Dubach et al. find that STZ volume has a clear positive dependence on strain rate at very low temperatures, but at ambient temperatures the data have too much variability to yield a strong dependence. Their positive STZ volume dependence at low temperatures matches our critical shear band nucleus size dependence but not our STZ volume dependence at ambient temperatures. This could be explained by the fact that they are likely measuring shear band nuclei sizes rather than STZ sizes in their acoustic emission tests [30]. On the other hand, Tönnies find that STZ volume has a negative correlation with strain rate at ambient temperatures [27], as does our model. But, they are also likely measuring shear band nuclei sizes which would contradict our positive strain rate dependence on shear band nuclei with strain rate. It could be that nanoindentation confines shear band nuclei sizes because larger and fewer shear bands might penetrate deep into the sample while profuse shear banding would involve smaller shear bands.

At this point, the exact physical basis of the STZ strain rate dependence is not clear. Dubach et. al. and Tönnies et. al. provide little discussion on the physical origin for the strain rate dependence they report $[27,30]$. However, one possible explanation is that the strain rate dependence comes from timedependent structural relaxations that occur in metallic glasses. For example, Argon's model for the STZ includes both the creation of free volume when an STZ is activated, as well as the redistribution of free volume through a competing diffusive process $[13,34]$. Atomistic simulations report a distribution of STZ events [39], which are altered under elastic [43] and plastic [44] loading. Specifically, these distributions 
of plastic events are altered to remove the low energy events [44]. Thus, one can reason that if these relaxation or redistribution events are time-dependent, at slower strain rates, there will be more time for relaxation to occur and remove these low energy events, leaving behind only the larger, higher energy events. At higher strain rates, there is less time for relaxation, so these lower energy events remain in the system. As a result, these could lead to an effective strain rate dependence on both the STZ volume and activation energy, both of which would decrease with increasing strain rate.

Alternatively, one could attribute the strain rate dependence to some other phenomena like local elastic properties [45] or local bonding such as icosahedral or non-icosahedral effects [46], that would also have a time-dependent relaxation associated with their evolution. In any case, it is noted that the variation of the energy barrier (1.08-1.68 eV) and the STZ volume $\left(1.6-2.6 \mathrm{~nm}^{3}\right)$ is small, and according to this work has log-linear dependence. These variations are likely within the resolution of techniques used to measure them. This functional dependence of these values may also explain the variation in reported measurements since different rates will result in different values. In the end, the experiments and the present simulations both indicate a rate dependence to the STZ behavior, which changes the critical nucleus size for shear banding. These minor changes lead to substantially different shear banding behaviors over the range of strain rates studied.

Finally, the critical nucleus size also has implications for improving ductility in metallic glasses. It is known that annealing/quenching and mechanical deformation can influence the ductility [47]; perhaps these can be used to influence the glass and increase the number of nucleation sites to the point that the glass is forced to deform in a more homogeneous manner. In addition, critical shear band nucleus size may also help explain why metallic glasses exhibit size-dependence for small samples. Metallic glass samples which are less than one $\mathrm{mm}$ in width are able to deform plastically more readily than larger samples; this is true for tension, compression, and bending [48-51]. Just as in the highest strain rate, where some shear bands traverse the simulation cell before achieving the critical nucleus size, perhaps 
the smaller experimental samples nucleate lots of shear bands because not enough dominant shear bands are able to nucleate.

\section{Conclusion}

The present work provides insight into the transition from strongly serrated flow at low strain rates to lightly serrated flow at high strain rates in metallic glasses deformed at ambient temperatures. The transition is investigated using the STZ dynamics framework, which now includes a strain rate dependence on the STZ volume and STZ activation energy. Shear band nucleation, propagation, sliding, and serration are examined statistically using 12 replicate simulations over 6 strain rates, ranging from $10^{-5} s^{-1}-10^{0} s^{-1}$

Statistical analysis of the results provides support for the hypothesis that the transition from strongly serrated flow to lightly serrated flow involves an increase of shear band nucleation rates and a decrease in shear band propagation and sliding speeds as strain rates increase. The magnitude of flow serration in the simulations appears to show transitions from strong to medium and medium to lightly serrated flow at the same rates predicted by Schuh's deformation map [6].

The present work hypothesizes that the underlying cause for this transition is a critical shear band nucleus size that increases with strain rate. At higher strain rates, it is more difficult to achieve nucleation so more shear bands are nucleated leading to less serrated flow. Interestingly, this critical nucleus size dependence emerges as a result of the fact that both STZ size and STZ activation energy decrease with increasing strain rate in the present model. This supports results from experiments, which also report rate dependent STZ properties $[27,30]$. While the variation in STZ size and activation energy are small, they have a significant effect on the shear banding behavior. Whether these STZ properties are rate dependent or are surrogates for other physical phenomena, such as free volume, they have 
implications for improving ductility in metallic glasses through the nucleation of additional shear bands to accommodate deformation. 
Acknowledgements: This work was supported by the National Science Foundation under Contract No.

CMMI-1401777.

\section{References}

[1] M. Telford. The case for bulk metallic glass, Materials Today 7 (2004) 36-43.

[2] W.H. Wang, C. Dong, C.H. Shek. Bulk metallic glasses, Materials Science and Engineering: R: Reports 44 (2004) 45-89.

[3] E.R. Homer, M.B. Harris, S.A. Zirbel, J.A. Kolodziejska, H. Kozachkov, B.P. Trease, J.-P.C. Borgonia, G.S. Agnes, L.L. Howell, D.C. Hofmann. New Methods for Developing and Manufacturing Compliant Mechanisms Utilizing Bulk Metallic Glass, Advanced Engineering Materials 16 (2014) 850-856.

[4] M.F. Ashby, A.L. Greer. Metallic glasses as structural materials, Scripta Materialia 54 (2006) 321326.

[5] C. Pampillo. Flow and fracture in amorphous alloys, J Mater Sci 10 (1975) 1194-1227.

[6] C.A. Schuh, T.C. Hufnagel, U. Ramamurty. Mechanical behavior of amorphous alloys, Acta Materialia 55 (2007) 4067-4109.

[7] C.A. Schuh, A.C. Lund, T.G. Nieh. New regime of homogeneous flow in the deformation map of metallic glasses: elevated temperature nanoindentation experiments and mechanistic modeling, Acta Materialia 52 (2004) 5879-5891.

[8] W.-D. Liu, K.-X. Liu, X.-X. Xia, W.-H. Wang. The failure stress of bulk metallic glasses under very high strain rate, Journal of Materials Research 25 (2010) 1230-1234.

[9] S.X. Song, H. Bei, J. Wadsworth, T.G. Nieh. Flow serration in a Zr-based bulk metallic glass in compression at low strain rates, Intermetallics 16 (2008) 813-818.

[10] F.H. Dalla Torre, D. Klaumünzer, R. Maaß, J.F. Löffler. Stick-slip behavior of serrated flow during inhomogeneous deformation of bulk metallic glasses, Acta Materialia 58 (2010) 3742-3750.

[11] C.A. Schuh, A.S. Argon, T.G. Nieh, J. Wadsworth. The transition from localized to homogeneous plasticity during nanoindentation of an amorphous metal, Philosophical Magazine 83 (2003) 2585-2597.

[12] W.H. Jiang, M. Atzmon. Rate dependence of serrated flow in a metallic glass, Journal of Materials Research 18 (2003) 755-757.

[13] A.S. Argon. Plastic deformation in metallic glasses, Acta Metallurgica 27 (1979) 47-58.

[14] E.R. Homer, D. Rodney, C.A. Schuh. Kinetic Monte Carlo study of activated states and correlated shear-transformation-zone activity during the deformation of an amorphous metal, Physical Review B 81 (2010) 064204.

[15] A.L. Greer, Y.Q. Cheng, E. Ma. Shear bands in metallic glasses, Materials Science and Engineering: R: Reports 74 (2013) 71-132.

[16] S. Ogata, F. Shimizu, J. Li, M. Wakeda, Y. Shibutani. Atomistic simulation of shear localization in Cu-Zr bulk metallic glass, Intermetallics 14 (2006) 1033-1037.

[17] F. Shimizu, S. Ogata, J. Li. Yield point of metallic glass, Acta Materialia 54 (2006) 4293-4298.

[18] D. Klaumünzer, R. Maaß, J.F. Löffler. Stick-slip dynamics and recent insights into shear banding in metallic glasses, Journal of Materials Research 26 (2011) 1453-1463.

[19] A.J. Cao, Y.Q. Cheng, E. Ma. Structural processes that initiate shear localization in metallic glass, Acta Materialia 57 (2009) 5146-5155.

[20] R.T. Qu, Z.Q. Liu, G. Wang, Z.F. Zhang. Progressive shear band propagation in metallic glasses under compression, Acta Materialia 91 (2015) 19-33.

[21] E.R. Homer. Examining the initial stages of shear localization in amorphous metals, Acta Materialia 63 (2014) 44-53. 
[22] S.X. Song, T.G. Nieh. Direct measurements of shear band propagation in metallic glasses - An overview, Intermetallics 19 (2011) 1968-1977.

[23] W.J. Wright, T.C. Hufnagel, W.D. Nix. Free volume coalescence and void formation in shear bands in metallic glass, Journal of Applied Physics 93 (2003) 1432-1437.

[24] B.P. Kanungo, S.C. Glade, P. Asoka-Kumar, K.M. Flores. Characterization of free volume changes associated with shear band formation in Zr- and Cu-based bulk metallic glasses, Intermetallics 12 (2004) 1073-1080.

[25] S.X. Song, X.L. Wang, T.G. Nieh. Capturing shear band propagation in a Zr-based metallic glass using a high-speed camera, Scripta Materialia 62 (2010) 847-850.

[26] I.-C. Choi, Y. Zhao, B.-G. Yoo, Y.-J. Kim, J.-Y. Suh, U. Ramamurty, J.-i. Jang. Estimation of the shear transformation zone size in a bulk metallic glass through statistical analysis of the first pop-in stresses during spherical nanoindentation, Scripta Materialia 66 (2012) 923-926.

[27] D. Tönnies, K. Samwer, P.M. Derlet, C.A. Volkert, R. Maaß. Rate-dependent shear-band initiation in a metallic glass, Applied Physics Letters 106 (2015) 171907.

[28] D. Rodney, A. Tanguy, D. Vandembroucq. Modeling the mechanics of amorphous solids at different length scale and time scale, Modelling and Simulation in Materials Science and Engineering 19 (2011) 083001.

[29] M. Zink, K. Samwer, W.L. Johnson, S.G. Mayr. Plastic deformation of metallic glasses: Size of shear transformation zones from molecular dynamics simulations, Physical Review B 73 (2006) 172203.

[30] A. Dubach, F.H. Dalla Torre, J.F. Löffler. Constitutive model for inhomogeneous flow in bulk metallic glasses, Acta Materialia 57 (2009) 881-892.

[31] L. Anand, C. Su. A constitutive theory for metallic glasses at high homologous temperatures, Acta Materialia 55 (2007) 3735-3747.

[32] E.R. Homer, C.A. Schuh. Mesoscale modeling of amorphous metals by shear transformation zone dynamics, Acta Materialia 57 (2009) 2823-2833.

[33] E.R. Homer, C.A. Schuh. Three-dimensional shear transformation zone dynamics model for amorphous metals, Modelling and Simulation in Materials Science and Engineering 18 (2010) 065009.

[34] L. Li, E.R. Homer, C.A. Schuh. Shear transformation zone dynamics model for metallic glasses incorporating free volume as a state variable, Acta Materialia 61 (2013) 3347-3359.

[35] J. Lu, G. Ravichandran, W.L. Johnson. Deformation behavior of the $\mathrm{Zr}_{41.2} \mathrm{Ti}_{13.8} \mathrm{Cu}_{12.5} \mathrm{Ni}_{10} \mathrm{Be}_{22.5}$ bulk metallic glass over a wide range of strain-rates and temperatures, Acta Materialia 51 (2003) 3429-3443.

[36] A.F. Voter. Introduction to the kinetic Monte Carlo method. Radiation Effects in Solids. Springer, 2007. pp. 1-23.

[37] X.L. Fu, Y. Li, C.A. Schuh. Homogeneous flow of bulk metallic glass composites with a high volume fraction of reinforcement, Journal of Materials Research 22 (2007) 1564-1573.

[38] J.B.C. Puthoff, H.B.; Jakes, Joseph E.; Voyles, P.M.; Stone, D.S. Shear transformation zone activation during deformation in bulk metallic glasses characterized using a new indentation creep technique. Materials Research Society symposium, vol. 1152. Warrendale, Pa.: Materials Research Society, 2009. p.6.

[39] D. Rodney, C. Schuh. Distribution of Thermally Activated Plastic Events in a Flowing Glass, Physical review letters 102 (2009) 235503.

[40] T.-H. Chen, C.-K. Tsai. The Microstructural Evolution and Mechanical Properties of Zr-Based Metallic Glass under Different Strain Rate Compressions, Materials 8 (2015) 1831.

[41] H. Kimura, T. Masumoto. A model of the mechanics of serrated flow in an amorphous alloy, Acta Metallurgica 31 (1983) 231-240.

[42] D. Jang, J.R. Greer. Transition from a strong-yet-brittle to a stronger-and-ductile state by size reduction of metallic glasses, Nat Mater 9 (2010) 215-219. 
[43] C. Deng, C.A. Schuh. Atomistic mechanisms of cyclic hardening in metallic glass, Applied Physics Letters 100 (2012) 251909.

[44] F. Delogu. Thermal and mechanical activation of inelastic events in metallic glasses, Scripta Materialia 113 (2016) 145-149.

[45] M. Tsamados, A. Tanguy, C. Goldenberg, J.-L. Barrat. Local elasticity map and plasticity in a model Lennard-Jones glass, Physical Review E 80 (2009) 026112.

[46] T. Egami. Atomic level stresses, Progress in Materials Science 56 (2011) 637-653.

[47] W.H. Jiang, M. Atzmon. Room-temperature flow in a metallic glass - Strain-rate dependence of shear-band behavior, Journal of Alloys and Compounds 509 (2011) 7395-7399.

[48] H. Guo, P.F. Yan, Y.B. Wang, J. Tan, Z.F. Zhang, M.L. Sui, E. Ma. Tensile ductility and necking of metallic glass, Nat Mater 6 (2007) 735-739.

[49] R.D. Conner, Y. Li, W.D. Nix, W.L. Johnson. Shear band spacing under bending of Zr-based metallic glass plates, Acta Materialia 52 (2004) 2429-2434.

[50] J.C. Huang, J.P. Chu, J.S.C. Jang. Recent progress in metallic glasses in Taiwan, Intermetallics 17 (2009) 973-987.

[51] G. Kumar, A. Desai, J. Schroers. Bulk Metallic Glass: The Smaller the Better, Advanced Materials 23 (2011) 461-476.

[52] W.L. Johnson, K. Samwer. A universal criterion for plastic yielding of metallic glasses with a ( $\mathrm{T} / \mathrm{Tg}$ ) 2/3 temperature dependence, Physical review letters 95 (2005) 195501.

[53] Q. Wang, J.M. Pelletier, J.J. Blandin, M. Suéry. Mechanical properties over the glass transition of $\mathrm{Zr}_{41.2} \mathrm{Ti}_{13.8} \mathrm{Cu}_{12.5} \mathrm{Ni}_{10} \mathrm{Be}_{22.5}$ bulk metallic glass, Journal of Non-Crystalline Solids 351 (2005) 2224-2231. 
Table 1: Material properties for modeling Vitreloy $1\left(\mathrm{Zr}_{41.2} \mathrm{Be}_{22.5} \mathrm{Ti}_{13.8} \mathrm{Cu}_{12.5} \mathrm{Ni}_{10}\right)$.

\begin{tabular}{|l|l|l|}
\hline Model Parameter & Name & Value \\
\hline STZ strain & $\gamma_{0}$ & 0.1 \\
\hline Shear modulus & $\mu_{0}$ & $35.76 \mathrm{GPa}[52]$ \\
\hline Poisson's ratio & $\mathrm{v}$ & $0.352[52]$ \\
\hline Debye temperature & $\theta_{\mathrm{D}}$ & $327 \mathrm{~K} \mathrm{[53]}$ \\
\hline
\end{tabular}


Figure 1: General deformation map for metallic glasses as a function of homologous temperature and applied strain rate, adapted from Schuh [6]. Points investigated in this paper are marked with a ' + ' on the deformation map.

Figure 2: Yield strength dependence on strain rate, before (red triangles) and after (blue diamonds) adjusting STZ volume and activation energy. After adjustment, the yield strength has no dependence on strain rate.

Figure 3: (a) Partial raw simulation result. (b) Individual shear bands identified with surrounding lines of unique color. (c) Shear band dominance measure by the fraction of all STZ activity occurring in each shear band over time; unique colors match those in (b). (d) Propagation velocity of shear band front, corresponding to the unique color in (b). (e) Sliding velocity profile of the primary shear band, identified in blue in (b), with velocity bursts labeled 1, 2, and 3. (f) Points on the stress-strain curve corresponding to labels 1, 2, and 3 in (e), showing the stress drops resulting from sliding events on the active shear band. (g) Snapshots of the simulation at times immediately following the bursts corresponding to labels 1,2 , and 3 in (e).

Figure 4: Overview of results. (a) Example simulation at each strain rate, where the number of shear bands in each simulation is near the median for that strain rate. Note the clear trend of increased shear band density with strain rate, and the increased appearance of free STZs at higher strain rates. (b) Stress-strain curves for the six simulations shown in (a). 
Figure 5: Box plot of the average stress drop magnitude in each simulation, arranged by strain rate. This indicates decreased flow serration with increasing strain rate, matching the deformation map in Figure 1.

Figure 6: (a) Plot of the nucleation rate after being normalized by strain rate. Inset shows the nucleation rate measured in shear band nuclei generated per second, on a log scale with a power-law fit. The normalized rate shows a positive correlation between nucleation rate and strain rate. (b) Shear band critical nucleus size plotted against strain rate. The skewed box plots in the lower strain rates are due to the cutoff size of detectable critical shear band nucleus size being reached.

Figure 7: (a) The maximum shear band propagation rate for each simulation, defined as the speed at which a shear band nucleus grows across a simulation, normalized by strain rate. (inset) Propagation speeds before normalization with a power-law fit. (b) The maximum shear band sliding rate for each simulation, defined as the relative velocity between points above and below the most dominant shear band, normalized by strain rate. (inset) Sliding speeds before normalization with a power-law fit.

Figure 8: Example simulations from the (a) slowest $\left(10^{-5} \mathrm{~s}^{-1}\right)$ and (b) fastest $\left(10^{0} \mathrm{~s}^{-1}\right)$ strain rates. These demonstrate the extreme differences in shear band propagation, where in (a), the shear band propagates in bursts only. In (b), the shear band has bursts in sliding, but always maintains a continuous sliding rate, though it is noted that most simulations at the highest strain rate did not have a dominant band this pronounced. The insets plot the sliding speed as a function of KMC steps to indicate how 
sliding appears from the perspective of individual STZ activations rather than the duration between activations.

Figure 9: Comparison of median values of the normalized shear band nucleation rate (yellow), shear band propagation rate (blue), and shear band sliding rate (green). The transition from propagation and sliding dominated shear banding at low strain rates to nucleation dominated shear banding at high strain rates supports the hypothesis that the transition in flow serration is a competition between these two stages. The increasing critical nucleus size (red) as a function of strain rate is proposed as the likely cause of this transition. 
Figure 1

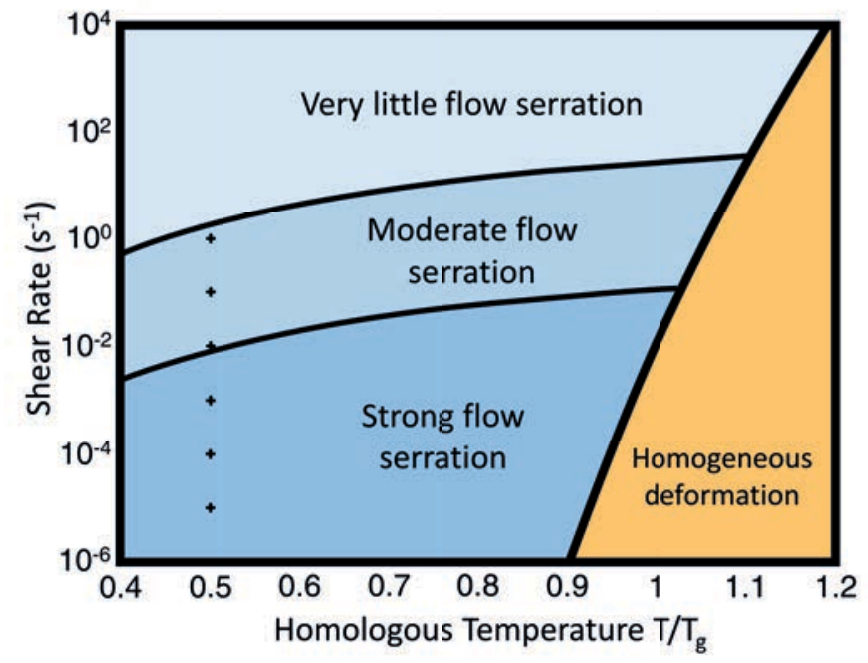


Figure 2

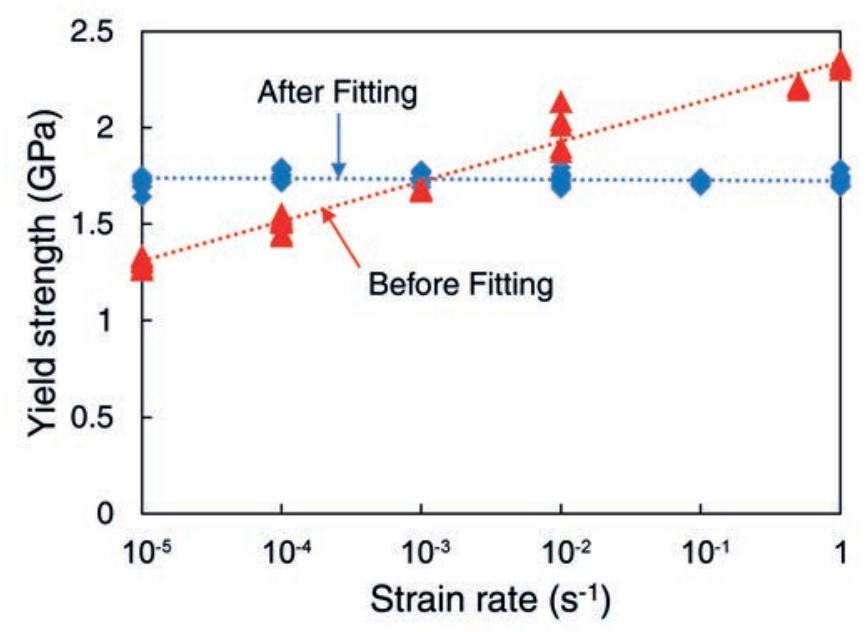


Figure 3

a

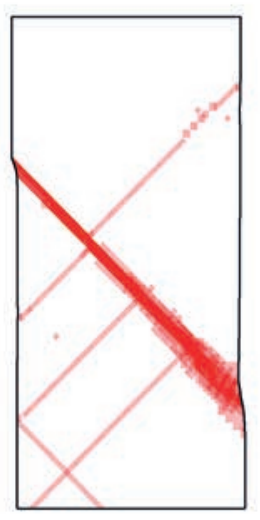

b

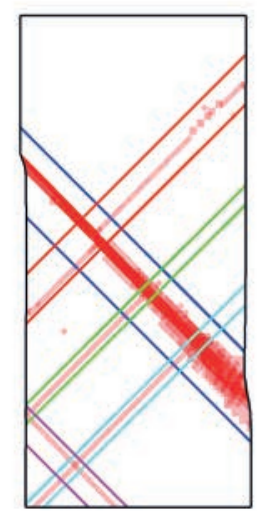

c

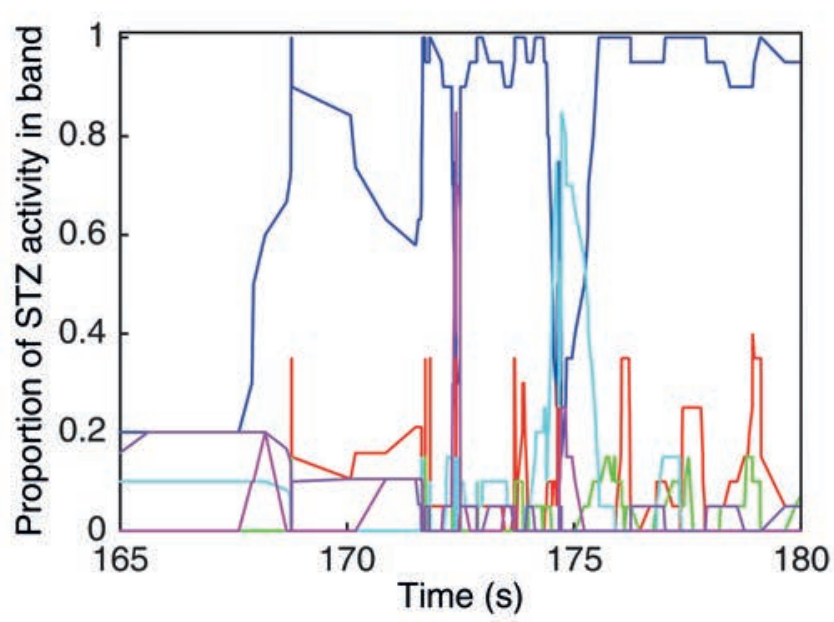

e

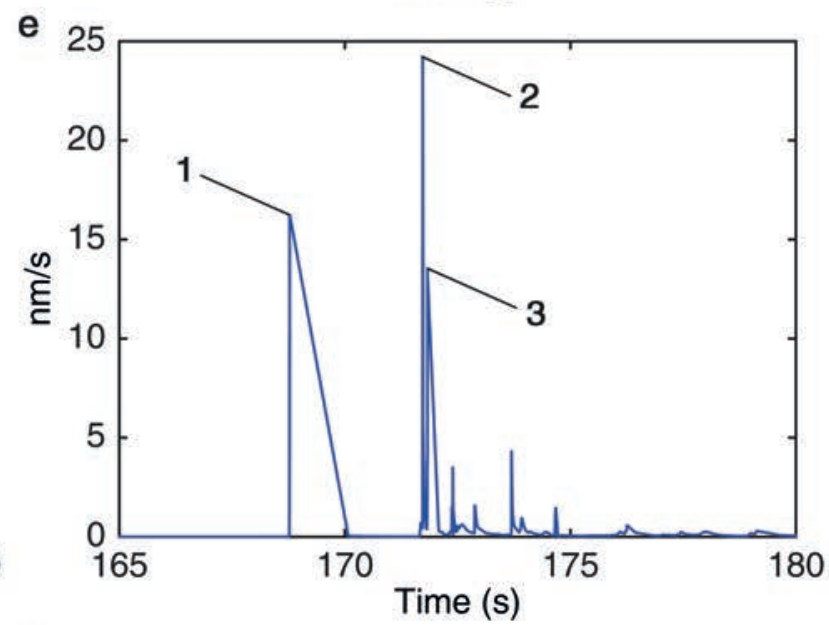

d
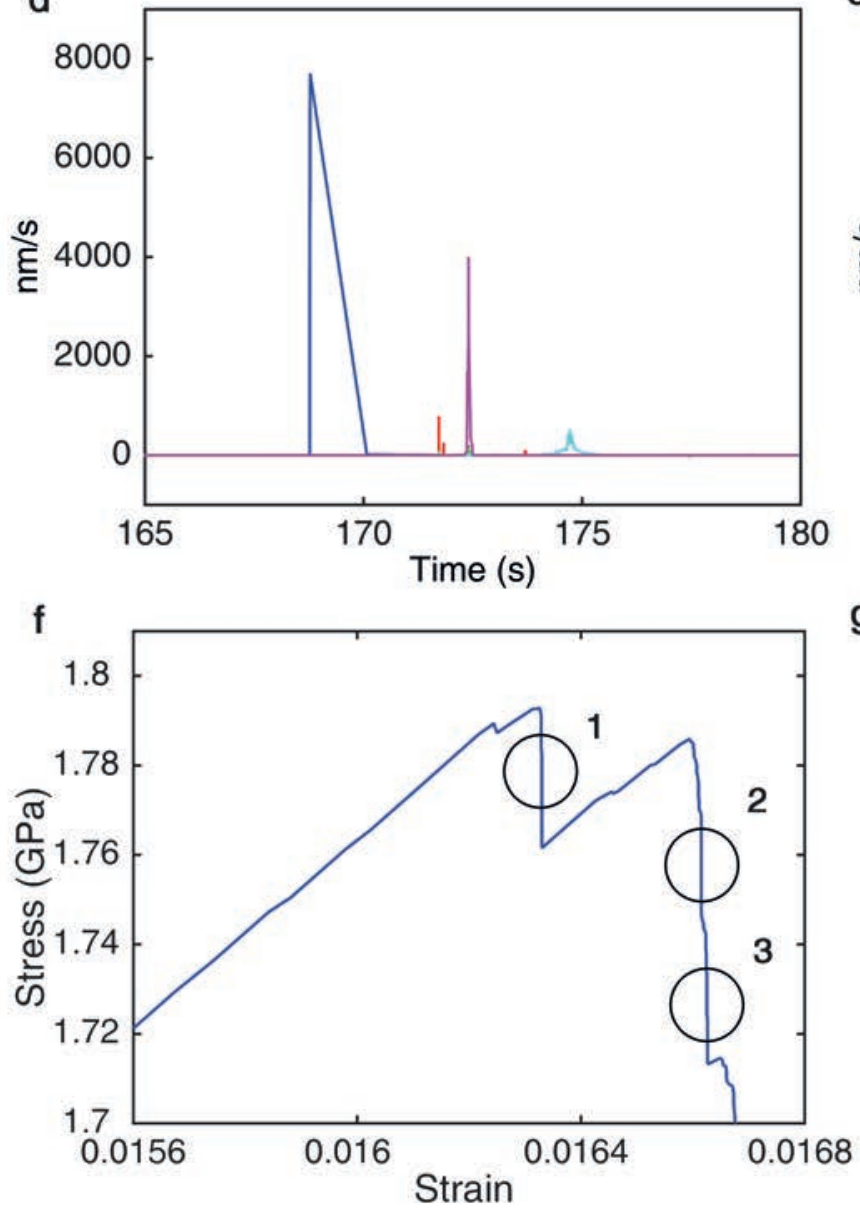

g

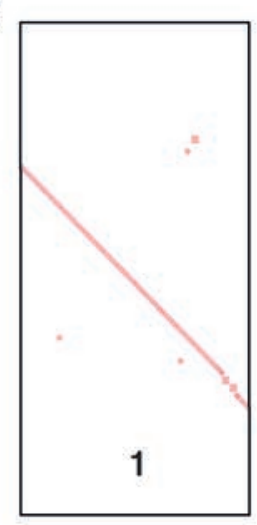

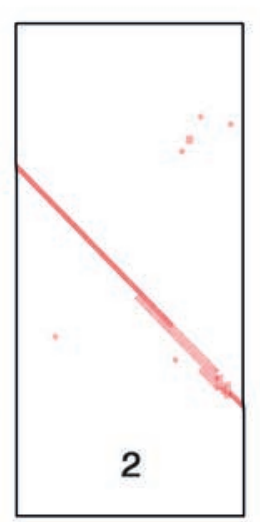

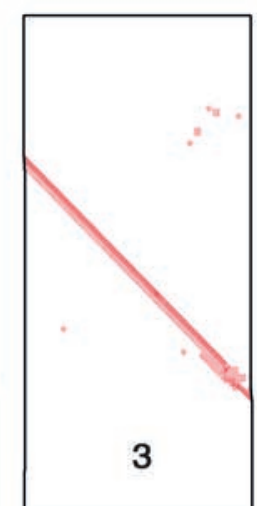


Figure 4
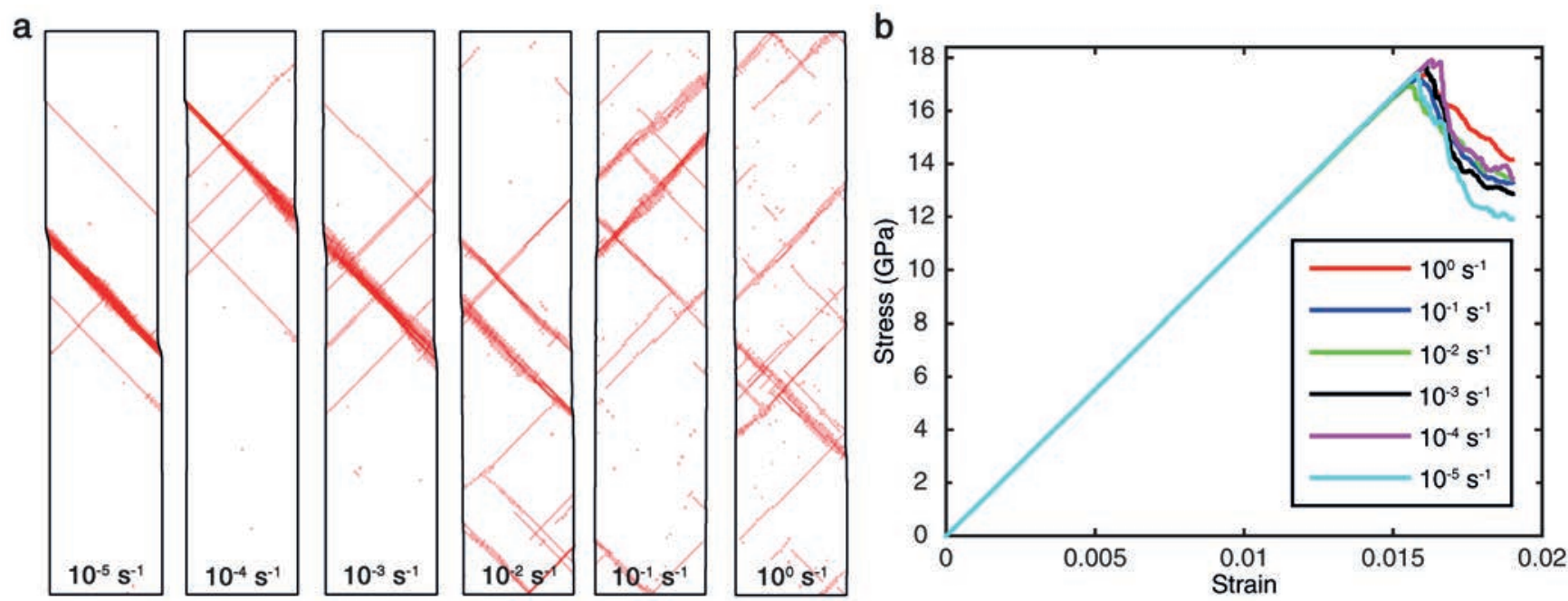
Figure 5

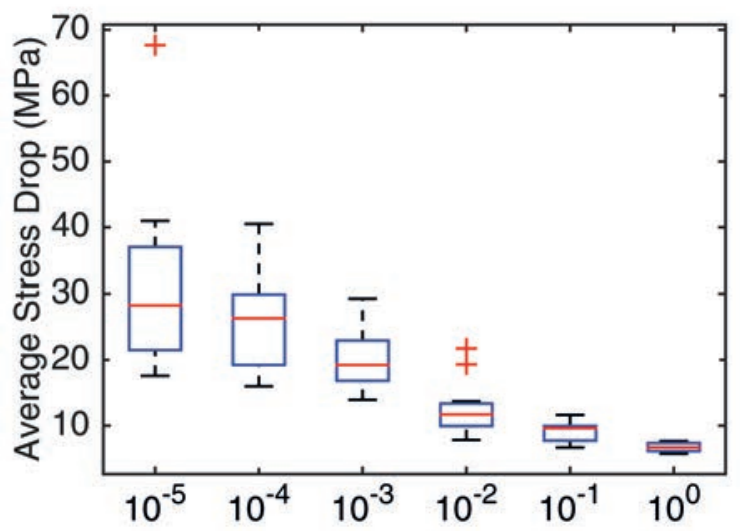


Figure 6
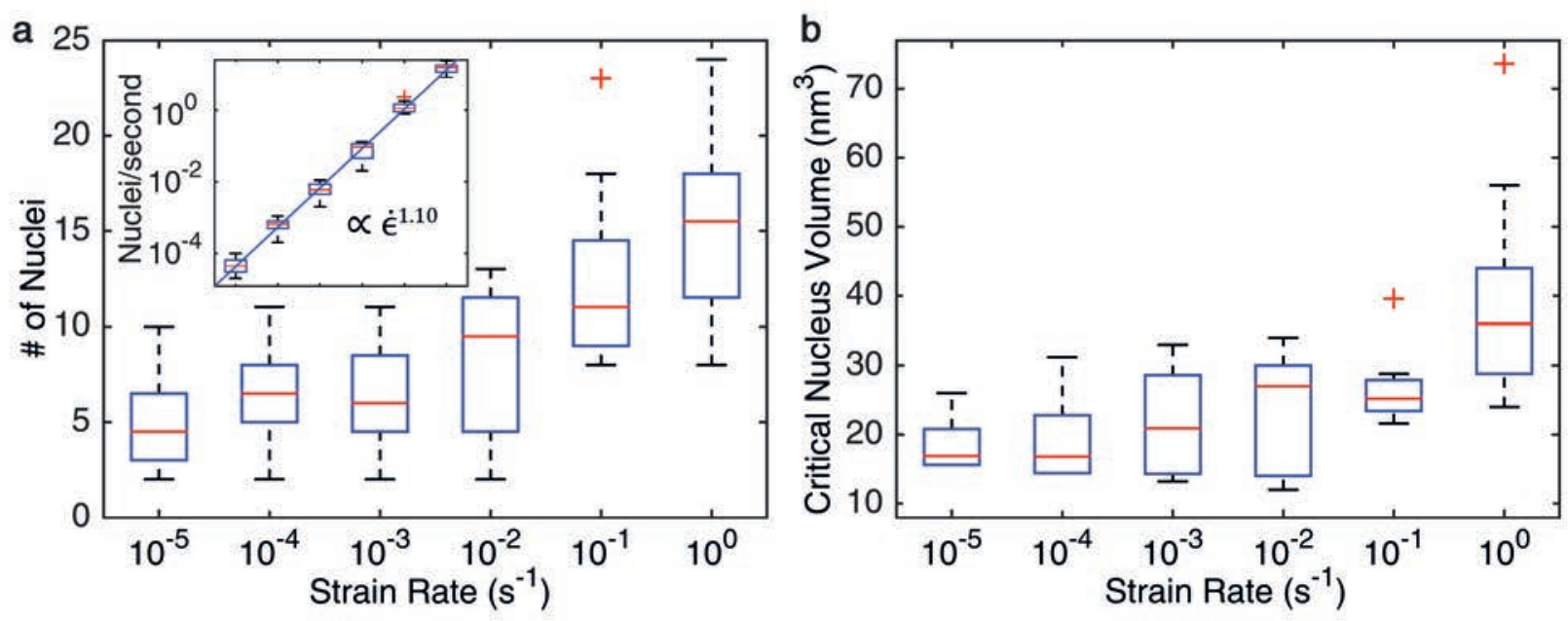
Figure 7
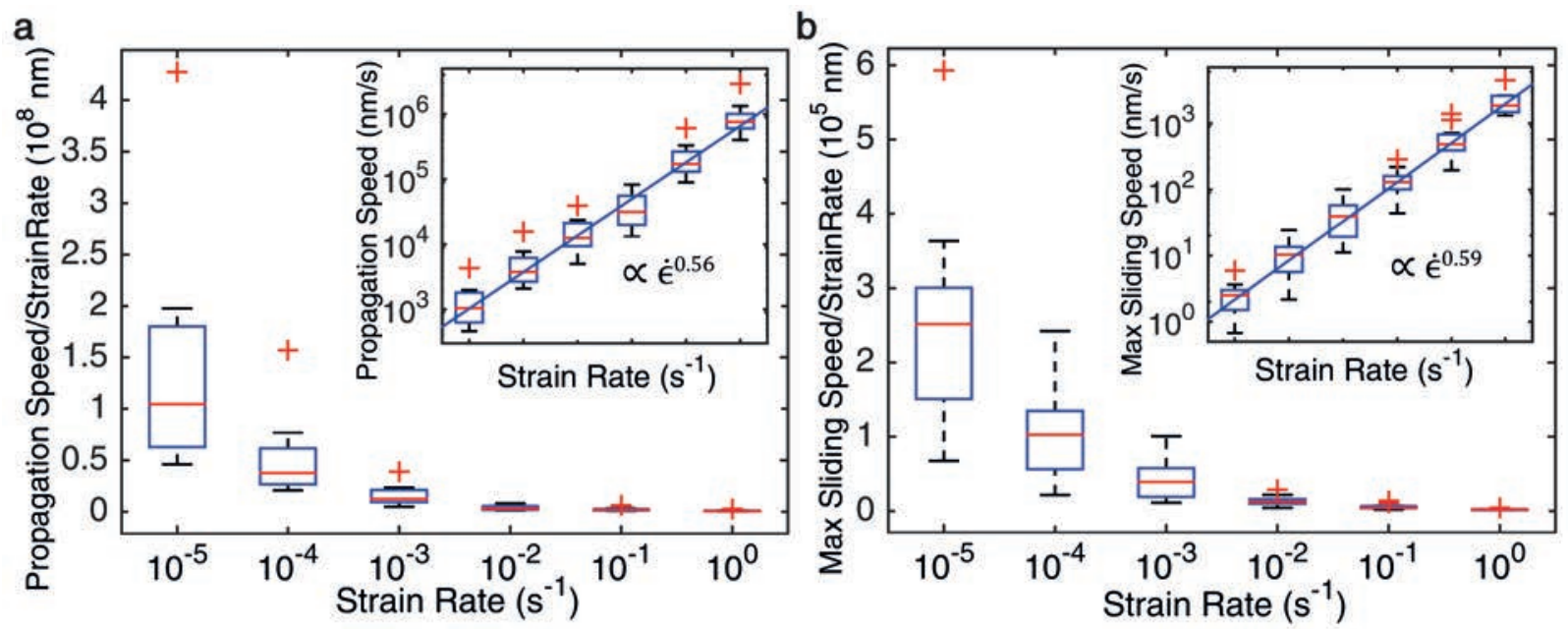
Figure 8

a

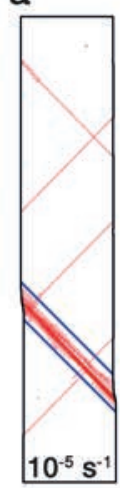

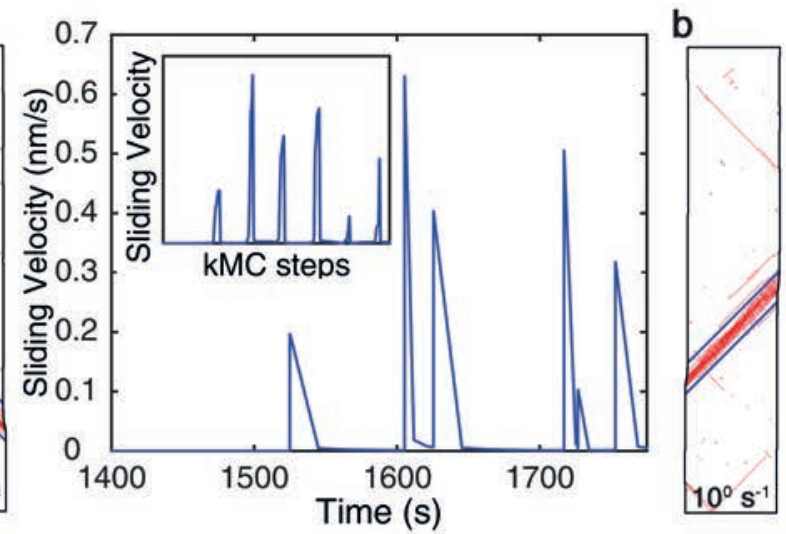

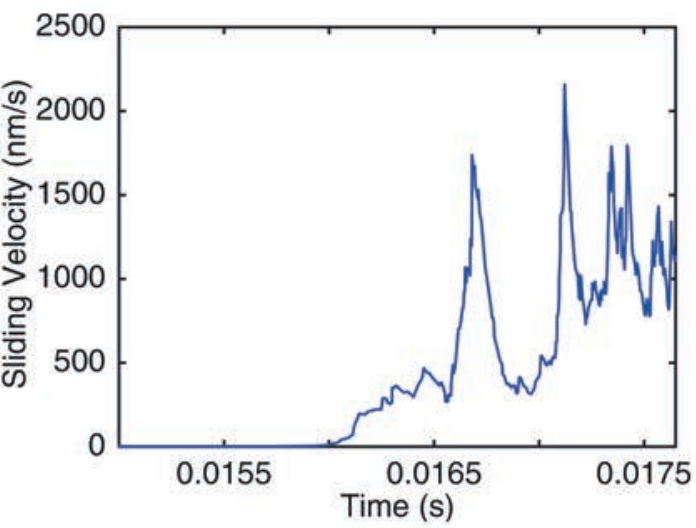


Figure 9

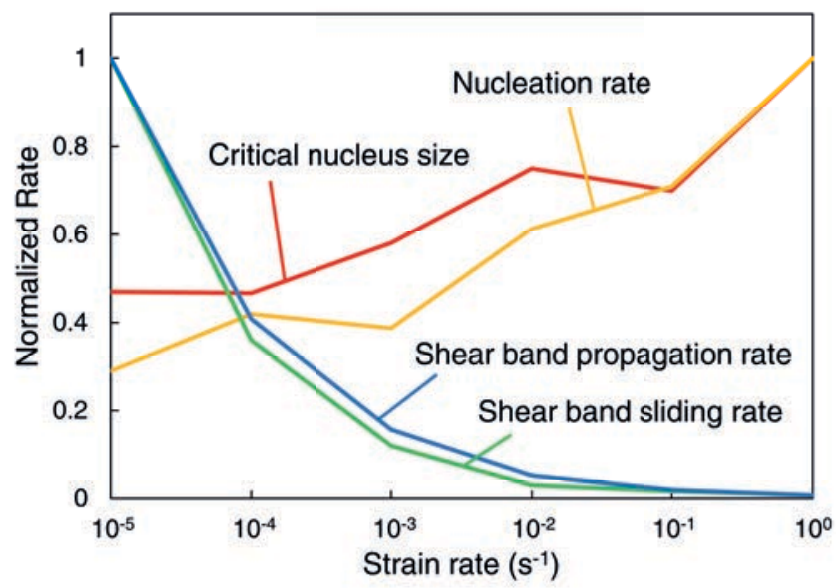

